\title{
Interleukin-12-anchored exosomes increase cytotoxicity of T lymphocytes by reversing the JAK/STAT pathway impaired by tumor-derived exosomes
}

\author{
YAO ZHANG ${ }^{1}$, XIAO-HOU WU ${ }^{1}$, CHUN-LI LUO ${ }^{2}$, JIA-MO ZHANG $^{1}$, BAI-CHENG HE ${ }^{3}$ and GANG CHEN ${ }^{1}$ \\ ${ }^{1}$ Department of Urology, The First Affiliated Hospital, Chongqing Medical University, Yi Xue Yuan Road, Chongqing; \\ ${ }^{2}$ Department of Laboratory Medicine; ${ }^{3}$ Pharmaceutical College, Chongqing Medical University, \\ Yi Xue Yuan Road, Chongqing, P.R. China
}

Received December 17, 2009; Accepted January 20, 2010

DOI: 10.3892/ijmm_00000393

\begin{abstract}
Tumor-derived exosomes express tumor antigens, leading to their promising utility as tumor vaccines, but they also can suppress $\mathrm{T}$-cell signaling molecules and reduce cytotoxic effects. We investigated whether interleukin-12 (IL-12)-anchored exosomes (EXO/IL-12) reverse tumor exosome-mediated inhibition of T-cell activation and cytotoxicity was associated with inhibition of JAK3 and p-STAT5. A co-expression plasmid of pBudCE4.1/IL-12A/ IL-12B-GPI was constructed. EXO/IL-12 was identified by transmission electron microscopy and Western blotting, which induced proliferation and cytotoxicity of T-cells and were analyzed by CFSE-based flow cytometry. Expression of JAK2, JAK3 and p-STAT5 was detected by Western blotting. Our results showed that EXO/IL-12 was much more efficient in induction of the proliferation, release of IFN- $\gamma$ and cytotoxic effect of $\mathrm{T}$ lymphocytes than conventional exosomes in vitro. Exosomes inhibited the expression of JAK3 and phosphorylation of STAT5 in high doses in T-cells, but not JAK2, while EXO/ IL-12 had much less attenuated reduction of the expression of p-STAT5. The enhanced cytotoxic effects of T lymphocytes might partly depend on EXO/IL-12 reversing the suppressed expression of p-Stat5 by Jak2/Stat5 pathway. These findings might provide an alternative approach for developing exosomes into tumor vaccines.
\end{abstract}

\section{Introduction}

One general characteristic of tumor cells is their ability to release exosomes. Exosomes $\left(\mathrm{EXO}_{\mathrm{S}}\right), 30-100 \mathrm{~nm}$ microvesicles secreted by many types of cells, have not been extensively

Correspondence to: Dr Xiao-Hou Wu, Department of Urology, The First Affiliated Hospital, Chongqing Medical University, Yi Xue Yuan Road, Chongqing, P.R. China

E-mail: wuxiaohou80@hotmail.com

Key words: exosomes, interleukin-12, signal transduction, tumor immunology investigated until Raposo et al for the first time, reported that B-cell-derived exosomes induced antigen-specific MHC class I-restricted T-cell responses in 1996 (1). The biology of exosomes derived from tumor cells $\left(\mathrm{TEX}_{\mathrm{S}}\right)$ has recently raised much interest due to their tumor rejection antigens, such as, MHC-I molecules, tetraspanins, HSP70-80, LAMP1 and ICAM-1, and serving as direct vaccines or processed by autologous dendritic cells, which can then be used to stimulate an anti-tumor response (2-6). As a cell-free vaccine, exosomes exhibit promising clinical applications. However, a recent study has shown that tumor-derived exosomes might be an alternative novel mechanism of immune escape because they express immunosuppressive molecules. These immunosuppressive molecules, FasL and TGF- 31 , are presently being linked to a series of functional alternations occurring in Tcells of patients with cancer, ranging from reduction of IFN- $\gamma$ production (7-9) to defects in T-cell receptor components and function, such as CD3- $\zeta$ and the downregulated JAK3STAT5 signal pathway (10-12). Therefore, a novel strategy should be explored to enhance the immunogenicity of exosomes and reverse the suppressed function of T-cells.

Interleukin-12 (IL-12) is a critical cytokine produced by phagocytic cells and antigen-presenting cells (APCs), and it plays a central role in the initiation and regulation of cellmediated immune responses through exerting a number of effects on T and NK cells (13). IL-12 promotes the killing capacity of NK and cytotoxic T-cells, drives the differentiation of Th0 to Th1 and strongly induces IFN- $\gamma$ secretion in T-cells (14). In addition, IL-12 can regulate the function of T-cell by the JAK/STAT signal pathway. Previous studies have indicated that IL-12 has the capacity to induce/maintain STAT4 and STAT5, and that TYK2 activation is associated with STAT4 phosphorylation leading to IFN- $\gamma$ induction, while JAK2 activation correlates with STAT5 phosphorylation and cellular proliferation (15-17). However, the potential use of IL-12 in clinical practice has been hampered by its very short half-life and severe side effects of systemic administration (18). The protein transfer method is an efficient approach to deliver cytokines at the immunization site and express the desired cytokines. Glycolipid-anchor-IL-12 (GPI-IL-12), could be effective in inducing proliferation and IFN- $\gamma$ production of T-cells (19-21). 
In the present study, we investigated whether EXO/IL-12 could reverse tumor exosome-mediated inhibition of T-cell activation and cytotoxicity was associated with the inhibition of JAK3 and p-STAT5. For this purpose, we constructed a mammalian co-expression plasmid of pBudCE4.1/IL-12A/ IL-12B-GPI, and prepared EXO/IL-12 from the culture supernatants of the stable cell line. We found that exosomes derived from renal cancer cells markedly suppressed the expression of JAK3 and p-STAT5 in high doses in T lymphocytes, but not JAK2, while EXO/IL-12 derived from EXO/ IL-12 could significantly reverse the suppressed expression of p-STAT5 by activating the JAK2-STAT5 pathway and increased induction of proliferation, IFN- $\gamma$ production and cytotoxicity of T-cells. These findings might provide an alternative approach to develop exosomes as tumor vaccine.

\section{Materials and methods}

Cell line and reagents. Human renal cancer cell line RC-2 and Human TCCB cell line T24 were purchased from Shanghai Cell Bank (China). PCR product purification kit was purchased from Shanghai-Huashun Biotechnology Corp. (China). Restriction endonuclease, quick ligation kit, Primstar enzymes and other PCR reagents were purchased from Takara Corp. (Japan).

Plasmids. A mammalian co-expression plasmid of GPI-anchorIL-12 was constructed by subcloning p35 cDNA (IL-12A) in pBudCE4.1 at KpnI/XhoI sites and a fusion gene containing GPI-anchor signal sequence of hPLAP-1 and p40 cDNA (IL12B) in the same plasmid at BamHI/XbaI sites. Detailed procedures were carried out as previously described (21).

Transfection. RC-2 transfectants expressing GPI-IL-12 were established by transfecting pBudCE4.1/IL-12A/IL-12B-GPI plasmid by Lipofectamine ${ }^{\mathrm{TM}} 2000$. RC-2 cells were inoculated in special FBS RPMI-1640 medium until $80 \%$ cells were confluent, cells transfected with empty plasmid and nontransfected cells as controls. Stable cell line was screened by Zeocin. About $100 \mathrm{ml}$ of stable cell line culture supernatant was collected for exosome preparation.

Preparation and identification of exosomes. The RC-2 cellderived exosomes were isolated and purified as described previously (21). Culture supernatants $(100 \mathrm{ml})$ were collected and obtained a volume of $\sim 3 \mathrm{ml}$ by ultrafiltration and sucrose $/ \mathrm{D}_{2} \mathrm{O}$ density gradient centrifugation. Membrane filter $(0.22 \mu \mathrm{m})$ was used for sterilization and the exosome was stored at $-80^{\circ} \mathrm{C}$. Bradford method was used for quantification of the total protein of exosomes. The morphology was identified by transmission electron microscopy. Pictures were analyzed with Digimizer Image Analysis Software (http://www.digimizer.com) to measure diameters of exosomes (EXO, exosomes derived from untransfected cells; $\mathrm{EXO/IL-12,} \mathrm{exosomes} \mathrm{derived} \mathrm{from} \mathrm{transfected} \mathrm{cells).}$

Confocal laser scanning microscopy and flow cytometry analysis. Cells were fixed with $4 \%$ paraformaldehyde and washed with PBS three times and blocked with $3 \%$ fetal bovine serum for $30 \mathrm{~min}$ at room temperature (RT), then incubated with mouse anti-human IL-12 antibody overnight at $4^{\circ} \mathrm{C}$ followed by incubation with FITC labeled second antibody for $1 \mathrm{~h}$. The images were observed and obtained by confocal microscopy. Untransfected cells and empty plasmid transfected cells served as controls. The GPI-linkage of IL-12 was confirmed by washing down RC-2-GPI-IL-12 cells with phosphatidylinositol-specific phospholipase C (PI-PLC) for flow cytometry to detect the membrane-bound GPI-IL-12 expression.

T-cell culture and proliferation assays. Peripheral blood mononuclear cells (PBMCs) were isolated from normal healthy donor as previously described (22). T-cells were enriched from PBMC by plate adherency and isolated by Nylon Fiber Column T (Seebio Co., China). PHA-activated T-cells were prepared using $5 \mu \mathrm{g} / \mathrm{ml}$ PHA (Sigma, Japan) by the standard procedure. Carboxyl fluorescein succinimidyl ester (CFSE)-based proliferation assays of T-cells were performed as previously described (23). CFSE (Molecular Probes, Inc., USA) was added to the cell suspension at a final concentration of $2.5 \mu \mathrm{M}$ and incubated for $10 \mathrm{~min}$ at RT in the dark. Labeling was terminated by adding the same volume of $50 \%$ fetal calf serum and washed with sterile PBS and resuspended in RPMI-1640 medium at $1 \times 10^{6} / \mathrm{ml}$. Labeled cells (responders) were co-cultured with PBS (100 $\mu 1)$, EXO $(10 \mu \mathrm{g} / 100 \mu \mathrm{l}), \mathrm{IL}-12(80 \mathrm{pg} / 100 \mu \mathrm{l})$ or EXO/IL-12 (10 $\mu \mathrm{g} /$ $100 \mu \mathrm{l}$ ) for $48 \mathrm{~h}$ in four independent assays, respectively. Each group had triplicate wells. Cultured cells were washed and analyzed using a FACScan flow cytometer.

IFN- $\gamma$ release assay. PHA-activated T-cells $\left(1 \times 10^{6}\right)$ (not dyed with CFSE) were co-cultured with PBS $(100 \mu 1)$, EXO $(10 \mu \mathrm{g} / 100 \mu \mathrm{l}), \mathrm{IL}-12(80 \mathrm{pg} / 100 \mu \mathrm{l})$ or EXO/IL-12 $(10 \mu \mathrm{g} /$ $100 \mu \mathrm{l}$ ) for $48 \mathrm{~h}$ in four independent assays as described in the proliferation assay. Supernatant was harvested and analyzed for the release of IFN- $\gamma$ by sandwich ELISA (eBioscience, Inc, USA). Each group had triplicate wells.

Western blot analysis. Western blot analysis was used for characteristic molecules of exosomes and proteins expressed in T-cells treated with exosomes or EXO/IL-12 $(0 \mu \mathrm{g} / 100 \mu 1$, $5 \mu \mathrm{g} / 100 \mu \mathrm{l}, 10 \mu \mathrm{g} / 100 \mu \mathrm{l}, 20 \mu \mathrm{g} / 100 \mu \mathrm{l})$. Exosomal protein was extracted as described (24) and T-cell lysates were boiled in SDS sample buffer. Total protein $(50 \mu \mathrm{g})$ was loaded into each well of SDS-gel for separation by PAGE, and then transferred onto PVDF membranes. Blotted membranes were blocked for $60 \mathrm{~min}\left(4^{\circ} \mathrm{C}\right)$ with TBST $(0.25 \mathrm{M}$ Tris, $\mathrm{pH} 7.5$, $150 \mathrm{mM}$ sodium chloride, and $0.2 \%$ Tween-20) containing $5 \%$ skim milk, followed by probing overnight with primary antibody and horseradish peroxidase coupled secondary antibody. After three washes with TBST, blotted proteins were detected using an image acquisition and analysis system. The following antibodies were used for the Western blots: anti-HSP70, anti-ICAM-1, anti-IL-12, anti-Jak3, antiJak2 and p-Stat5 antibodies (Santa Cruz, USA).

Cytotoxicity assays. Dendritic cells were isolated from PBMC for cytotoxicity assay. Adhered cells from PBMC were propagated in RPMI-1640 complete medium containing $1,000 \mathrm{IU} / \mathrm{ml}$ GM-CSF and IL-4 for 6 days. The morphology of DC was identified by SEM. Exosomes loaded DCs promoted activation of autologous T-cells used as effector cells. Target T-cells were RC-2. Autologous DCs and T-cells 
A

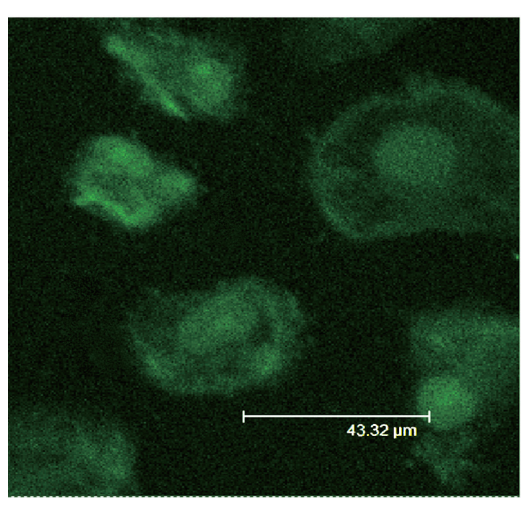

B

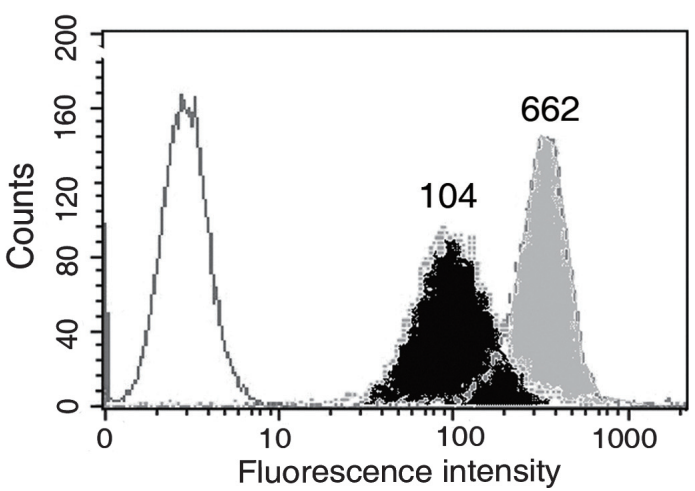

Figure 1. (A) Confocal laser scanning microscopy observated the expression of GPI-IL-12 on cell surface of stable transfectants. Cells were fixed with $4 \%$ paraformaldehyde, and immunostained with mouse anti-human IL-12 antibody overnight at $4^{\circ} \mathrm{C}$, followed by FITC labeled second antibody for $1 \mathrm{~h}$ at $37^{\circ} \mathrm{C}$, bars, $43.32 \mu \mathrm{m}$. (B) Chimeric GPI-IL-12 was expressed as GPI-anchored form. Cells were immunostained with anti-IL-12 mAb (filled histogram), followed by FITC labeled second antibody. PI-PLC-treated cells were analyzed by flow cytometry. The values for mean fluorescent intensity for IL-12 expression were indicated. PI-PLC-treated cells showed lower levels of cell surface expression of GPI-IL-12.
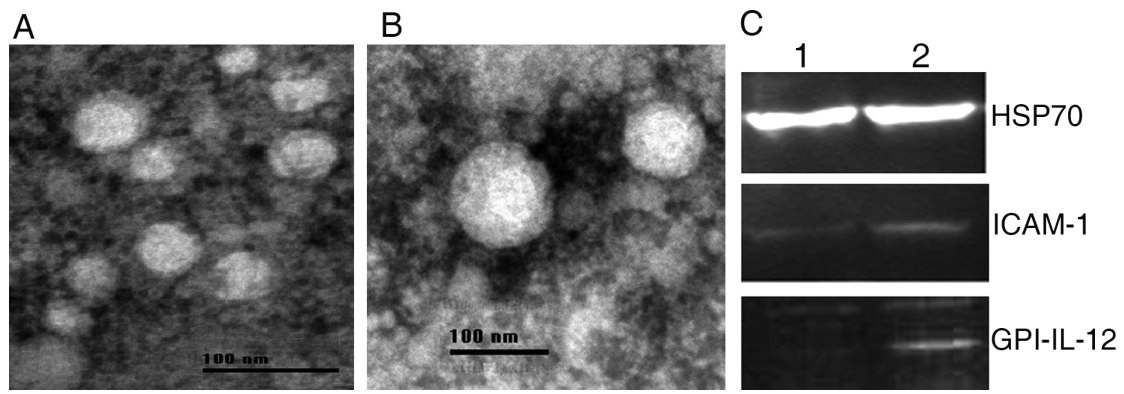

Figure 2. Identification of tumor-derived exosomes. Exosomes were isolated from RC-2 cells transfected with fusion gene or from RC-2 cells without gene transfection, and then visualized under TEM. They were spherical vesicles surrounded by the two-layer lipid membrane. The mean diameter was $52.2 \pm 25.6$ nm and $95 \%$ of them distributed between 30-80 nm. (A) Exosomes derived from gene-modified renal cancer cells; bar, $100 \mathrm{~nm}$. (B) Conventional exosomes, bar, $100 \mathrm{~nm}$. (C) Western blotting analyzed protein compositions of exosomes. Equal amount of exosomal protein derived from 1 (non transfeced group), 2 (transfected group), the primary antibodies used were anti-HSP70, anti-ICAM-1 and anti-IL-12 antibodies.

co-inoculated with EXO/IL-12 $(10 \mu \mathrm{g} / 100 \mu \mathrm{l})$ for $48 \mathrm{~h}$ in a humidified $5 \% \mathrm{CO}_{2}$ incubator. IL-12, EXO was used as control. Cytotoxicity assays based on PI co-staining of CFSE-labeled target cells were performed precisely as described (25). In brief, target cells were labeled with $2.5 \mu \mathrm{M}$ CFSE, which strongly labels cells and allows differentiation between target and effector cells. Labeled target cells $\left(10^{4}\right)$ were co-incubated in 24-well plates with given amounts of effector cells for $6 \mathrm{~h}$ in a humidified $5 \% \mathrm{CO}_{2}$ incubator. All cells were harvested and resuspended in PBS and analyzed by flow cytometry. Each group had triplicate wells. Killing rate was assessed by the percentage of PI costaining cells out of gated CFSElabeled cells. In all experiments the percentage of PI-positive CFSE-labeled cells in wells cultured without effectors did not exceed $15 \%$. Specific killing rate $=($ experimental killing rate - natural death rate) / (100\% - natural death rate); experimental killing rate $=\left(\mathrm{CFSE}^{+} / \mathrm{PI}^{+}\right.$target cells $) /\left(\mathrm{CFSE}^{+}\right.$target cells $)$.

Statistical analysis. Statistical differences between two groups were analyzed by one-way analysis of variance (ANOVA) or Student's t-test. Data were recorded as mean $\pm \mathrm{SD}$, and values of $\mathrm{P}<0.05$ considered significant.

\section{Results}

IL-12 was anchored on cell surfaces as a GPI-anchored protein. IL-12 was mainly expressed on the membrane of the stable transfectants of RC-2 cells and also observed on the membrane of the nucleus. No fluorescence intensity was observed in the non-transfected and empty vector groups (data not shown) (Fig. 1A). To determine whether the chimeric IL-12 on the cell surface was expressed as GPI-anchored protein, RC-2-GPI-IL-12 cells were treated with PI-PLC and analyzed by flow cytometry. PI-PLC-treated cells showed lower levels of cell surface expression of GPI-IL-12, indicating that IL-12 was anchored to cell surfaces via GPI-moiety (Fig. 1B).

EXO/IL-12 prepared from the RC-2-GPI-IL-12 transfectants. Exosomes derived from RC-2-GPI-IL-12 cells were successfully isolated. Transmission electron microscopy (TEM) showed that exosomes were spherical vesicles with a mean $52.2 \pm 25.6 \mathrm{~nm}$ and surrounded by a two-layer lipid membrane. Exosomes $(95 \%)$ had a diameter $30-80 \mathrm{~nm}$. The mean protein concentration of exosomes was $1.7 \pm 0.26 \mathrm{mg} / \mathrm{ml}$. There were no significant differences in morphology and size 

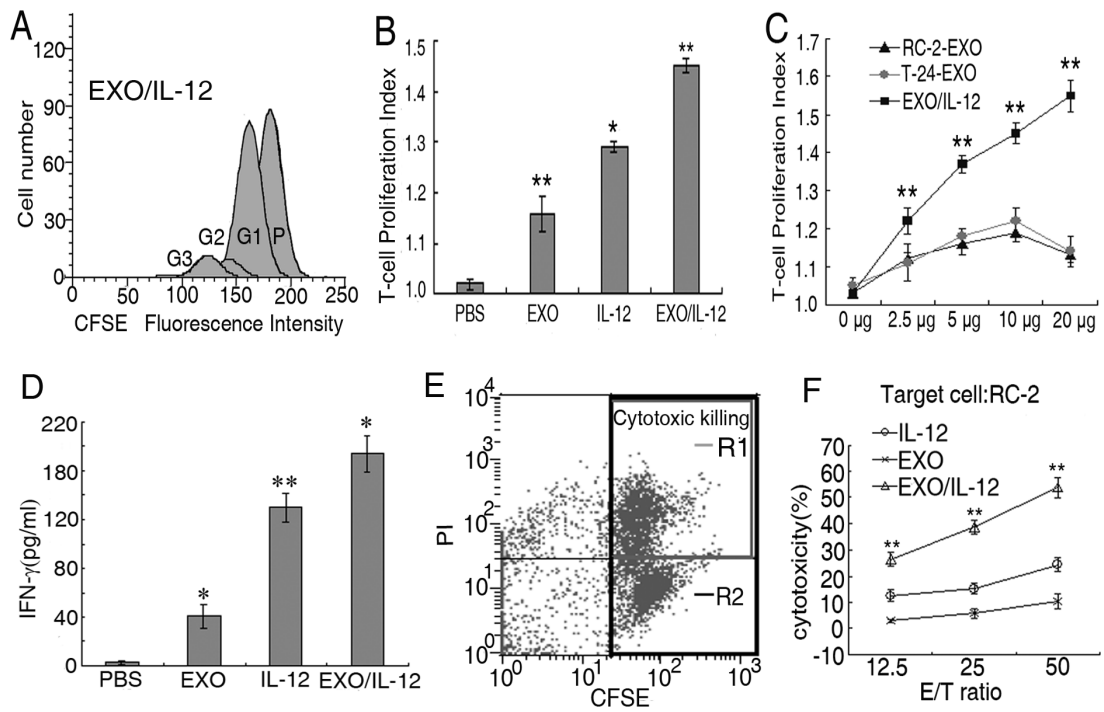

Figure 3. EXO/IL-12 induces proliferation and IFN- $\gamma$ of T-cells. (A) PHA-activated T-cells were stained with CFSE $(2.5 \mu \mathrm{M})$ for 10 min at $37^{\circ} \mathrm{C}$ and resuspended into RPMI-1640 medium at a concentration of $1 \times 10^{6}$ cells $/ \mathrm{ml} /$ well in 24-well plates, followed by stimulation of PBS, EXO (10 $\left.\mu \mathrm{g}\right)$, IL-12 (80 pg) or EXO/IL$12(10 \mu \mathrm{g})$ for $48 \mathrm{~h}$ in four independent assays. Labeled cells were collected and analyzed for the proliferation rate by FACScan flow cytometry. P, parent cells; G1, G2, G3, generation 1, 2, 3, respectively. (B) T-cell proliferation index was analysed with stimulation of PBS, EXO, IL-12 and EXO/IL-12. (C) T-cell proliferation index was detected with stimulation at varying concentrations of RC-2-EXO, T-24-EXO and EXO/IL-12. (D) EXO/IL-12 induced the release of IFN- $\gamma$ by T-cells. PHA activated T-cells co-cultured with PBS, EXO, IL-12 or EXO/IL-12, respectively. Supernatants were collected after $48 \mathrm{~h}$ and the release of IFN- $\gamma$ was determined by ELISA. EXO compared with PBS; IL-12 compared with EXO; EXO/IL-12 compared with IL-12. (E and F) Increased induction of CTL activity by stimulation of EXO/IL-12. (E) A two-color flow cytometric assay measured cytotoxicity of tumor cells by effector cells. Lymphocytes were excluded by setting gated. Region 1 (R1) contained target cells killed externally by effector cells (cytotoxicity) and were therefore $\mathrm{CFSE}^{+} / \mathrm{PI}^{+}$. Region 2 (R2) represents total CFSE ${ }^{+}$ tumor cell targets. Killing rate $=\left(\mathrm{CFSE}^{+} / \mathrm{PI}^{+}\right.$target cells $) /\left(\mathrm{CFSE}^{+}\right.$target cells $)$. (F) Lymphocytes were isolated from PBMCs and co-incubated with EXO, IL-12 or EXO/IL-12 for $48 \mathrm{~h}$. Stimulated T-cells used as effectors, and target cells were RC-2. Cytotoxicity assays based on PI co-staining of CFSE-labeled target cells were performed at varying effector-to-target cell ratios. Values are presented as mean $\pm \mathrm{SD}$ of triplicate wells. ${ }^{*} \mathrm{P}<0.05,{ }^{* *} \mathrm{P}<0.01$.

among those exosomes derived from RC-2 cells or RC-2GPI-IL-12 cells. To testify whether GPI-IL-12 expressed on exosomes were derived from the RC-2-GPI-IL-12 transfectants and whether GPI-IL-12 influenced the protein components of exosomes, we compared the protein pattern of exosomes derived from cells of two groups (transfected and nontransfected groups) by Western blotting as shown in Fig. 2 . GPI-IL-12 was detected in exosomes in the transfection group, but not in the other. There was no significant difference among the protein expression of HSP70, ICAM-1 on exosomes derived from cells of the two groups. The amount of IL-12 was $80 \pm 9.6 \mathrm{pg} / \mathrm{ml}$ in $10 \mu \mathrm{g}$ of exosomes, quantified by ELISA assay. These results showed that exosome-GPI-IL-12 can be obtained from IL-12-anchored renal cancer cells and IL-12 did not influence the protein components of exosomes.

EXO/IL-12 enhance proliferation, IFN- $\gamma$ release and cytotoxic effects of T-cells. To determine whether EXO/IL-12 induces proliferation of activated T-cells more efficiently, we measured the proliferation rate of activated T-cells stimulated with PBS, EXO, IL-12 or EXO/IL-12, respectively, in a CFSEbased proliferation assay by flow cytometry. As shown in Fig. 3A-C. The proliferative response of T-cells was significantly enhanced by stimulation with EXO, IL-12 or EXO/IL-12 alone compared with PBS. The highest proliferating reaction was observed in T-cells stimulated with EXO/IL-12 group. Interestingly, EXO/IL-12-mediated proliferation of activated T-cells in a dose-dependent manner while tumor-derived exosomes inhibited this proliferative response in high doses $(20 \mu \mathrm{g} / 100 \mu \mathrm{l})$. IL-12 has been shown to induce the release of Th1 type cytokines such as IFN- $\gamma$ (4). Therefore, the ability of EXO/IL-12 to induce the release of IFN $-\gamma$ was determined in an IFN- $\gamma$ release assay by ELISA. The results demonstrated that EXO, IL-12 and EXO/IL-12 could induce lymphocytes to secrete IFN- $\gamma$. The amount of IFN- $\gamma$ was $174.3 \pm 14.6 \mathrm{pg} / \mathrm{ml}$ secreted by lymphocytes induced by EXO/IL-12, which was more than that in the two other groups $(\mathrm{P}<0.05)$ (Fig. 3D). To explore whether EXO/IL-12 could elicit more powerful cytotoxic effect, T-cells were stimulated by EXO, IL-12 or EXO/IL-12 loaded autologous dendritic cells for $48 \mathrm{~h}$, respectively, in five independent assays. Activated T-cells used as effector cells were added to corresponding targets at varying effector-to-target cell ratios. The results showed that T-cells initiated by EXO/IL-12 kill tumor cells more efficiently than by the EXO and IL-12 groups $(\mathrm{P}<0.01)$ (Fig. 3F). Collectively, the results indicate that EXO/IL-12 were more powerful in inducing T-cell proliferation, IFN- $\gamma$ release and cytotoxic effects than conventional exosomes.

EXO/IL-12 reversed inhibition of the JAK/STAT pathway impaired by tumor exosomes. Previous data suggested that expression of JAK3 and p-STAT5 were inhibited by tumorderived exosomes (11). To determine whether EXO/IL-12 reverses tumor exosome-mediated inhibition of T-cell activation and if cytotoxicity was associated with inhibition of JAK3 and p-STAT5, the expression of JAK3 and p-STAT5 was quantified by Western blot analysis. We found that the expression of Jak3 (Fig. 4A, top panel) and p-Stat5 (Fig. 4A, second panel), but not JAK2 (Fig. 4A, third panel), were inhibited by the RC-2-derived exosomes in high doses 

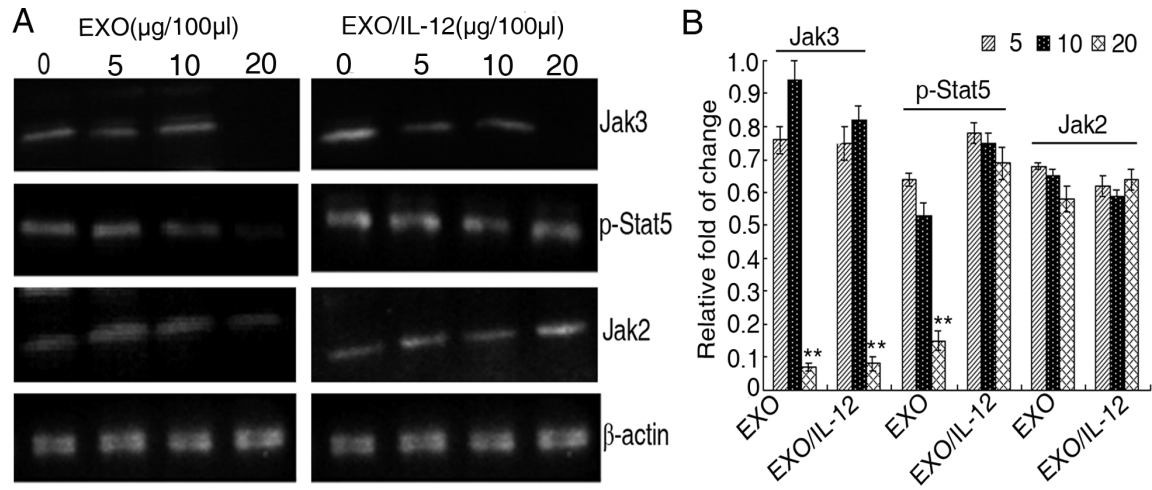

Figure 4. EXO/IL-12 reversed the inhibition of p-STAT5 expression impaired by tumor-derived exosomes. (A) T-cells were cultured in the presence of different concentrations of $\mathrm{EXO}_{\mathrm{s}}$ and EXO/IL-12 for $48 \mathrm{~h}$. T-cells were lysed. Total protein $(50 \mu \mathrm{g})$ was loaded into each well of an SDS-gel for separation by PAGE, and then transferred onto PVDF membranes. Blots were probed with the indicated antibodies. (B) Signal intensity of each protein analyzed was quantified using Quantity One-4.6.2 (Bio-Rad). Data are representative of three independent experiments. ${ }^{* *} \mathrm{p}<0.01$.

$(20 \mu \mathrm{g} / \mathrm{ml})$. In contrast, EXO/IL-12 had much less attenuated reduction of the expression of p-STAT5, but it had the same inhibition effect on JAK3 as exosomes. Exosome or EXO/ IL-12 had no effect on the expression of JAK2. Further quantification of expression of p-STAT5 indicated that EXO/ IL-12 can significantly reverse the expression of p-STAT5 impaired by tumor-derived exosomes ( $\mathrm{p}<0.01$ ) (Fig. 4B). The difference in the amount of phosphorylated STAT5 among samples might be attributed to GPI-IL-12 which reversed the expression of p-STAT5 by the JAK2-S STAT5 signal pathway.

\section{Discussion}

Exosomes represent a population of membrane vesicles that are homogeneous in size (ranging from 60 to $100 \mathrm{~nm}$ ) and shape. They are formed initially by inward budding of the limiting membrane into the lumen of endosomes, creating multi-vesicular endosomes. The exosomes are most likely secreted into the extracellular environment upon fusion of the multi-vesicular endosomes with the plasma membrane (26). Exosomes do not mirror the general composition of the plasma membrane of the originating tumor cell, but represent 'micromaps', with enhanced expression of tumor antigens (27). With high expression of immune associated protein, such as MHC-I, molecules, co-stimulatory molecules, heat shock protein70, and intercellular adhesion molecules, exosomes serve as direct or indirect vaccines to stimulate the anti-tumor response in many animal tumor models and result in significant inhibition of tumor growth $(3,4)$. As a cell-free vaccine, exosomes avoid many shortcomings of traditional cancer vaccines and are promising for tumor vaccines. However, clear evidence has accumulated showing that these exosomes can indeed exert an array of unfavorable effects on different crucial components of the anti-tumor immune response $(7-12,28)$. These data show that tumor-derived exosomes can (a) induce apoptosis in activated T-cells, through pathways used by the immune system itself to downsize immune reactions; (b) switch off natural cytotoxic responses, such as those mediated by natural killer cells; (c) reduce the release of IFN- $\gamma$ and proliferation of T-cells by suppressing the expression of CD3- $\zeta$ and downregulating the JAK3-STAT5 signal pathway. Although the precise mechanism is poorly understood, ongoing efforts should be focused on developing novel strategy to enhance the immunogenicity of exosomes and reverse the impaired signal pathway in order to induce a more efficient anti-tumor response.

In this study, we confirmed that exosomes prepared from IL-12-anchored renal cancer cells also expressed GPI-IL-12. Firstly, the stable transfectants can be obtained from GPI-IL-12 gene-modified renal cancer cells and IL-12 was expressed on the cell surface via a GPI-moiety by confal laser scanning microscopy and flow cytometry. Secondly, IL-12 ELISA and Western blot analysis demonstrated that the fusion protein was also anchored on the exosomes derived from culture supernatants of IL-12 anchored RC-2 cells. The amount of IL-12 was $80 \pm 9.6 \mathrm{pg} / \mathrm{ml}$ in $10 \mu \mathrm{g}$ of exosomes. The mechanism of GPI-IL-12 spontaneously anchored onto exosomes was not clear. A possible reason was that GPI-IL-12 had existed on the tumor cell membrane before exosomes formed by inward budding from the limiting cell membrane. Therefore, our results indicated that, by combining with the GPI anchor technology, EXO/IL-12 can be prepared from IL-12-anchored renal cancer cells. We have also investigated whether EXO/ IL-12 increases induction of proliferation, secretion of IFN- $\gamma$ of activated T-cells. As shown in Fig. 3A-D, EXO/IL-12 significantly induced higher levels of secretion of IFN- $\gamma$ and proliferation of activated T-cells as compared to exosomes and soluble IL-12. The reason for this difference between soluble IL-12 and EXO/IL-12 in induction of IFN- $\gamma$ production is not clear. It is possible that EXO/IL-12 efficiently provided much more activated signals (such as co-stimulatory signals) to induce the proliferation of T-cells and release of IFN- $\gamma$ compared to soluble IL-12. More importantly, EXO/IL-12 exhibited a much more potent anti-tumor effect than conventional exosomes and IL-12 in vitro, as shown in (Fig. 3E and F). Therefore, our results indicated that, by combining with the GPI anchor technology and EXO/IL-12 was more powerful in inducing T-cell proliferation, IFN- $\gamma$ release and cytotoxic effects than conventional exosomes.

Previous data suggested that the expression of both CD3- $\zeta$ and JAK3 protein were inhibited by tumor-derived exosomes, and further led to reduction of phosphorylation level of STAT5 $(11,12)$. In this study, we found that renal cancer-derived exosomes also downregulated the expression of JAK3 and of 
phosphorylation STAT5 in high doses in T lymphocytes, but not JAK2. In contrast, EXO/IL-12 had much less attenuated reduction of the expression of p-STAT5, but it had the same inhibition effect on JAK3 as exosomes. Interestingly, exosome or EXO/IL-12 had no effect on the expression of JAK2 in $\mathrm{T}$ lymphocytes. Therefore, EXO/IL-12-increased induction of cytotoxicity of $\mathrm{T}$ lymphocytes might partly depend on upregulating the expression of p-STAT5. The mechanism of EXO/IL-12 which upregulated the expression of p-STAT5 was not clear. Possible reasons were that (a) the two JAK family members, JAK2 and TYK2, were activated when IL-12 was combined with its receptor (IL-12R). Activation of JAKs led to the tyrosine phosphorylation of a family of STATs. STAT5 was one of substrates of JAK2 and JAK3 $(15,29,30)$. (b) IL-12 has the capacity to induce/maintain STAT4 and STAT5, and TYK2 activation is associated with STAT4 phosphorylation leading to IFN- $\gamma$ induction, while JAK2 activation correlates with STAT5 phosphorylation and cellular proliferation (16). Therefore, the results suggested that EXO/ IL-12 could phosphorylate STAT5 by an alternative approach of JAK2/STAT5 signal pathway when the JAK3/STAT5 pathway was suppressed by tumor-derived exosomes in $\mathrm{T}$ lymphocytes.

In conclusion, our data confirmed that EXO/IL-12 was much more efficient in induction of the proliferation, release of IFN- $\gamma$ and cytotoxic effect of T lymphocytes than conventional exosomes in vitro. The enhanced cytotoxic effects of $\mathrm{T}$ lymphocytes might partly be dependent on EXO/IL-12 reversing the suppressed expression of p-Stat 5 by Jak $2 /$ Stat 5 pathway when the Jak3/Stat5 pathway was suppressed by tumor-derived exosomes. These findings might provide an alternative approach for developing exosomes for tumor vaccines.

\section{Acknowledgements}

We thank Professor T.C. He (Department of Molecular Oncology, Chicago University) for his gift of pAdTraceTO4IL-12B-GPI plasmid and helpful advice.

\section{References}

1. Raposo G, Nijman HW, Stoorvogel W, Liejendekker R, Harding CV, Melief CJ and Geuze HJ: B lymphocytes secrete antigen-presenting vesicles. J Exp Med 183: 1161-1172, 1996.

2. Andre F, Schartz NE, Movassagh M, et al: Malignant effusions and immunogenic tumor-derived exosomes. Lancet 360: 295-305, 2002.

3. Chaput N, Taïeb J, Schartz NE, André F, Angevin E and Zitvogel L: Exosome-based immunotherapy. Cancer Immunol Immunother 53: 234-239, 2004.

4. Wolfers J, Lozier A, Raposo G, et al: Tumor-derived exosomes are a source of shared tumor rejection antigens for CTL crosspriming. Nat Med 7: 297-303, 2001.

5. Théry C, Zitvogel L and Amigorena S: Exosomes: composition, biogenesis and function. Nat Rev Immunol 2: 569-579, 2002.

6. Dai S, Wan T, Wang B, et al: More efficient induction of HLAA0201-restricted and carcinoembryonic antigen (CEA)-specific CTL response by immunization with exosomes prepared from heat-stressed CEA-positive tumor cells. Clin Cancer Res 11: 7554-7563, 2005.

7. Andreola G, Rivoltini L, Castelli C, et al: Induction of lymphocyte apoptosis by tumor cell secretion of FasL-bearing microvesicles. J Exp Med 195: 1303-1316, 2002.

8. Huber V, Fais S, Iero M, et al: Human colorectal cancer cells induce T-cell death through release of proapoptotic microvesicles: role in immune escape. Gastroenterology 128: 17961804,2005
9. Kim JW, Wieckowski E, Taylor DD, Reichert TE, Watkins S, and Whiteside TL: Fas ligand-positive membranous vesicles isolated from sera of patients with oral cancer induce apoptosis of activated T lymphocytes. Clin Cancer Res 11: 1010-1020, 2005.

10. Zhang HG, Kim H, Liu C, et al: Curcumin reverses breast tumor exosomes mediated immune suppression of NK cell tumor cytotoxicity. Biochim Biophys Acta 1773: 1116-1123, 2007.

11. Taylor DD and Gerçel TC: Tumour-derived exosomes and their role in cancer-associated T-cell signalling defects. Br J Cancer 92: 305-311, 2005.

12. Taylor DD, Gerçel TC, Lyons KS, Stanson J and Whiteside TL: T-cell apoptosis and suppression of T-cell receptor/CD3- $\zeta$ by Fas ligand-containing membranous vesicles shed from ovarian tumor. Clin Cancer Res 9: 5113-5119, 2003.

13. Trinchieri G: Interleukin-12: a cytokine produced by antigenpresenting cells with immunoregulatory functions in the generation of T-helper cells type 1 and cytotoxic lymphocytes. Blood 84: 4008-4027, 1994.

14. Colombo MP and Trinchieri G: Interleukin-12 in anti-tumor immunity and immunotherapy. Cytokine Growth Factor Rev 13: 155-168, 2002.

15. Gollob JA, Murphy EA, Mahajan S, Schnipper CP, Ritz J and Frank D: Altered interleukin-12 responsiveness in Th1 and Th2 cells is associated with the differential activation of STAT5 and STAT1. Blood 91: 1341-1354, 1998.

16. Ahn HJ, Tomura M, Yu WG, Iwasaki M, Park WR, Hamaoka T, and Fujiwara H: Requirement for distinct janus kinases and STAT proteins in T cell proliferation versus IFN- $\gamma$ production following IL-12 stimulation. J Immunol 161: 5893-5900, 1998.

17. Morales VA, Smits HH, Cantrell DA and Hilkens CM: Sustained IL-12 signaling is required for Th1 development. J Immunol 172: 61-69, 2004.

18. Sangro B, Melero I, Qian C and Prieto J: Gene therapy of cancer based on interleukin 12. Current Gene Therapy 5: 573-581, 2005.

19. Nagarajan S and Selvaraj P: Glycolipid-anchored IL-12 expressed on tumor cell surface induced antitumor immune response. Cancer Res 62: 2869-2874, 2002.

20. Nagarajan S and Selvaraj P: Human tumor membrane vesicles modified to express glycolipid-anchored IL-12 by protein transfer induce $\mathrm{T}$ cell proliferation in vitro: A potential approach for local delivery of cytokines during vaccination. Vaccine 24 : 2264-2274, 2006.

21. Zhang Y, Luo C-L, He B-C, Zhang J-M and Cheng G, Wu X-H: Exosomes derived from IL-12-anchored renal cancer cells increase induction of specific antitumor response in vitro: a novel vaccine for renal cell carcinoma. Int J Oncol 36: 133-140, 2010.

22. Wang YC, Zhu L, McHugh R, et al: Induction of autologous tumor-specific cytotoxic T-lymphocyte activity against a human renal carcinoma cell line by B7-1 (CD80) costimulation. J Immunother 19: 1-8, 1996.

23. Liu DC, Yu J, Chen HY, Reichman R, Wu HL and Jin X: Statistical determination of threshold for cellular division in the CFSE-labeling assay. J Immunol Methods 312: 126-136, 2006.

24. Zitvogel L, Regnault A, Lozier A, et al: Eradication of established murine tumors using a novel cell-free vaccine: dendritic cell-derived exosomes. Nature Med 4: 594-600, 1998.

25. Karagiannis SN, Bracher MG, Hunt J, et al: IgE-antibodydependent immunotherapy of solid tumors: Cytotoxic and phagocytic mechanisms of eradication of ovarian cancer cells. J Immunol 179: 2832-2843, 2007.

26. Keller S, Sanderson MP, Stoeck A and Altevogt P: Exosomes: from biogenesis and secretion to biological function. Immunol Lett 107: 102-108, 2006.

27. Taylor DD and Black PH: Neoplastic and development importance of plasma membrane vesicles. Am Zool 26: 411-415, 1987.

28. Valenti R, Huber V, Filipazzi P, et al: Human tumor-released microvesicles promote the differentiation of myeloid cells with transforming growth factor-beta-mediated suppressive activity on T lymphocytes. Cancer Res 66 : 9290-9298, 2006.

29. Schindler C, Levy DE and Decker T: JAK-STAT signaling: from interferons to cytokines. J Biol Chem 282: 20059-20063, 2007.

30. Walker and Smith: The Jak-STAT pathway in rheumatoid arthritis. J Rheumatol 32: 1650-1653, 2005. 